\title{
Characterization of Stabilizing Switching Sequences in Switched Linear Systems Using Piecewise Linear Lyapunov Functions ${ }^{\star}$
}

\author{
Xenofon D. Koutsoukos ${ }^{1}$ and Panos J. Antsaklis ${ }^{2}$ \\ 1 Xerox Palo Alto Research Center \\ 3333 Coyote Hill Road, Palo Alto, CA 94304, USA \\ koutsouk@parc.xerox.com \\ 2 Department of Electrical Engineering \\ University of Notre Dame, Notre Dame, IN 46556, USA \\ antsaklis.1@nd.edu
}

\begin{abstract}
In this paper, the stability of switched linear systems is investigated using piecewise linear Lyapunov functions. Given a switched linear system, we present a systematic methodology for computing switching laws that guarantee stability based on the matrices of the system. We assume that each individual subsystem is stable and admits a piecewise linear Lyapunov function. Based on these Lyapunov functions, we compose "global" Lyapunov functions that guarantee stability of the switched linear system. A large class of stabilizing switching sequences for switched linear systems is characterized by computing conic partitions of the state space. The approach is applied to both discrete-time and continuous-time switched linear systems.
\end{abstract}

\section{Introduction}

In this paper, we study the stability of continuous and discrete-time switched linear systems using piecewise linear Lyapunov functions and we identify classes of switching sequences that result in stable trajectories. The main motivation behind this problem is that it is often easier to find switching controllers than to find a fixed controller. In the case when we have multiple control objectives, we may design a continuous controller for each control objective, and control the behavior of the plant by switching between different controllers. For example, in the control of the longitudinal dynamics of an aircraft with constrained angle of attack, the control objective is twofold: track the pilot's reference normal acceleration while maintaining a safety constraint in the angle of attack [8]. A continuous feedback control law can be easily designed for each control objective resulting in two asymptotically stable subsystems and a switching mechanism can be used to simultaneously achieve both objectives. Such a switching system might become unstable for certain switching sequences, even if all the individual

\footnotetext{
* The partial financial support of the National Science Foundation (ECS99-12458) and the Army Research Office (DAAG55-98-1-0199) is gratefully acknowledged.
} 
subsystem are stable (see for example [8]). For such problems, it is important to characterize switching sequences that result in stable trajectories.

Stability of switched systems has been studied extensively in the literature; see for example $[8,16,17]$ and the references therein. Sufficient conditions for uniform stability, uniform asymptotic stability, exponential stability and instability were established in [22]. Necessary conditions (converse theorems) for some of the above stability results have also been established. Analysis tools for switched and hybrid systems based on multiple Lyapunov functions were presented in [5]. Stability analysis of switched systems is usually carried out using a Lyapunov-like function for each subsystem [8]. These Lyapunov functions are pieced together in some manner in order to compose a Lyapunov function that guarantees that the energy of the overall system decreases to zero along the state trajectories of the system. The application of the theoretical results to practical hybrid systems is accomplished usually using a linear matrix inequality (LMI) problem formulation for constructing a set of quadratic Lyapunov-like functions [12, 21]. Existence of a solution to the LMI problem guarantees that the hybrid system is stable. However, in order to formulate the LMI problem, a partition of the state space and therefore a switching law must be known a priori. Usually, such a partition consists of a set of ellipsoidal regions derived by exploiting the physical insight for the particular application. Although, the LMI approach for hybrid system stability is computationally efficient, it is based only on sufficient conditions and more importantly, it relies on a particular partition chosen by the designer.

In order to investigate the stability properties of practical hybrid systems, there is an important need to characterize partitions of the state space that lead to stable trajectories based on the system parameters. Such partitions can be used very efficiently for the design of switching control laws that guarantee stability of the overall system. In our approach, we characterize a large class of switching sequences that result in stable trajectories. Given a switched linear system, we present a systematic methodology for computing switching laws based on the system parameters that guarantee stability. We assume that each individual subsystem is stable and admits a piecewise linear Lyapunov function. Based on these Lyapunov functions, we compose "global" Lyapunov functions that guarantee stability of the switched linear system. The main contribution of this work is that based on the piecewise linear Lyapunov functions we construct a conic partition of the state space that is used to characterize a large class of switching laws that result in stable trajectories.

It should be noted that the problem considered in this paper has been addressed using multiple Lyapunov function tools under the assumption that switching among stable systems is slow enough $[8,16]$. Here, we consider piecewise linear Lyapunov functions and we develop a systematic approach to characterize stabilizing switching sequence that offers a significant advantage. Individual piecewise linear Lyapunov functions are "pieced together" in a systematic way and they result in a conic partition of the state space that can be used very efficiently for the design of the switching control law. Note that the paper 
reports results from [14] and that early results for the discrete-time case have been reported in [15].

The paper is organized as follows. In Section 2, the problem of identifying stabilizing switching sequences is described. Section 3 presents the necessary background for piecewise linear Lyapunov functions. The emphasis is put on computational methods for constructing such Lyapunov functions. The technical results for the characterization of stabilizing switching sequences are presented in Section 4 . The application of the methodology to continuous-time switched linear systems is presented in Section 5. Finally, concluding remarks are presented in Section 6.

\section{Problem Statement}

In this section, we consider discrete-time switched linear systems described by

$$
x(t+1)=A_{q} x(t), \quad q \in Q=\{1, \ldots, N\}
$$

where $x(t) \in \Re^{n}, t \in \mathbf{Z}^{+}$(the set of nonnegative integers) and $A_{q} \in \Re^{n \times n}$.

The mathematical model described by (1) represents the continuous (state) portion of a piecewise linear hybrid dynamical system. The particular mode $q$ at any given time instant may be selected by a decision-making process. In this paper, we represent such a decision-making process by a switching law of the form

$$
q(t+1)=\delta(q(t), x(t)) .
$$

Given $x(t)$, the next state is computed using the mode $q(t)$, that is $x(t+1)=$ $A_{q(t)} x(t)$. The function $\delta: Q \times \Re^{n} \rightarrow \Re^{n}$ is discontinuous with respect to $x$. A switching law is defined here using a partition of the state space.

Our objective is to investigate the stability of the switched linear system (1) under the switching law (2). Note that the origin $x_{e}=0$ is an equilibrium for the system (1). Furthermore, for a particular switching law, the switched system (1) can be viewed as a special case of a time-varying linear system, and therefore the usual definitions of stability can be used; see for example [1].

\section{$3 \quad$ Piecewise Linear Lyapunov Functions}

In this section, we briefly present some background material necessary for the stability analysis of switched linear systems presented later in this paper. We consider the discrete-time linear system

$$
x(t+1)=A x(t)
$$

where $x(t) \in \Re^{n}$ and $A \in \Re^{n \times n}$.

Definition 1. A nonempty set $P \subset \Re^{n}$ is said to be (positively) invariant for the system (3) if $x(0) \in P$ implies that $x(t) \in P$ for every $t \in\left(\mathbf{Z}^{+}\right) \mathbf{Z}$. 
In the case when the system admits a positively invariant polyhedral set $P$ containing the origin a Lyapunov function can be constructed by considering the Minkowski functional (gauge function) of $P$; see for example [3]. For bounded invariant polyhedral sets this is accomplished as follows (the extension to unbounded polyhedral sets is straightforward):

Let $F_{i}$ be a face of a polytope and consider the corresponding hyperplane $H_{i}$ as shown in Fig. 1. The hyperplane can be described (perhaps after normalization) by $H_{i}=\left\{x \in \Re^{n}:\left\langle x, w_{i}\right\rangle=1\right\}$. where $w_{i} \in \Re^{n}$ is the gradient vector of the hyperplane and $\langle\cdot, \cdot\rangle$ denotes the inner product.

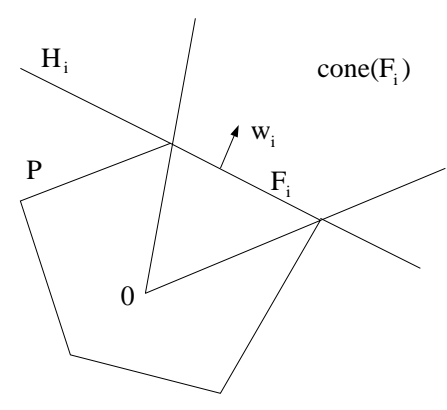

Fig. 1. A polytope $P$, a face $F_{i}$ and its corresponding hyperplane $H_{i}$.

Since the set $P$ includes an open neighborhood of the origin, $\Re^{n}$ can be partitioned into a finite number of cones defined as follows. Each face $F$ of the polytope can be described as the convex hull of its extreme points $f_{j} \in \Re^{n}, j=$ $1, \ldots, r$. A finitely generated cone can be defined for the face $F$ by

$$
\operatorname{cone}(F)=\left\{x \in \Re^{n}: x=\sum_{j-1}^{r} \alpha_{j} f_{j}, \alpha_{j}>0, j=1, \ldots, r\right\} .
$$

Consider a polytope $P \subset \Re^{n}$ and assume that $0 \in \operatorname{int}(P)$. The Minkowski functional of $P$ is defined by

$$
V(x)=\inf \{\rho>0 \mid x \in \rho P\}
$$

where $\rho P=\{\rho x \mid x \in P\}$. Consider a particular face $F_{i}$ and the corresponding cone. Since $F_{i} \in \partial P$ there exist unique $\rho>0$ and $\hat{x} \in F_{i}$ such that for any $x \in \operatorname{cone}\left(F_{i}\right)$ we have $x=\rho \hat{x}$ and the Minkowski functional can be computed by

$$
V(x)=\frac{\|x\|_{2}}{\|\hat{x}\|_{2}}=\rho=\rho\left\langle\hat{x}, w_{i}\right\rangle=\left\langle x, w_{i}\right\rangle
$$

since $\left\langle\hat{x}, w_{i}\right\rangle=1$. Therefore, for $x \in \operatorname{cone}\left(F_{i}\right)$, the Lyapunov function induced by the set $P$ can be written as $V(x)=\left\langle x, w_{i}\right\rangle$. Consequently, the Lyapunov function induced by $P$ can be computed for $x \in \Re^{n}$ by

$$
V(x)=\max _{1 \leq i \leq m}\left\langle x, w_{i}\right\rangle \text {. }
$$


A special case of piecewise linear Lyapunov functions arise when the positively invariant set $P$ of Definition 1 is centrally symmetric. In this case, the Lyapunov function $V(x)$ can be represented using the infinity norm. Furthermore, there exists a class of linear systems for which such a Lyapunov function can be computed very efficiently. Consider the following Lyapunov function candidate $V(x)=\|W x\|_{\infty}$ where $W \in \Re^{m \times n}$ and $\|\cdot\|_{\infty}$ denotes the infinity norm defined by $\|x\|_{\infty}=\max _{1 \leq i \leq n}\left|x_{i}\right|$.

Theorem 1. [2] $V(x)=\|W x\|_{\infty}$ is a Lyapunov function for the system (3) if and only if there exist a matrix $Q \in \Re^{m \times m}$ such that $W A-Q W=0$ and $\|Q\|_{\infty}<1$.

It should be noted that similar results have been established for differential and difference inclusions in [19].

\subsection{Computation of Piecewise Linear Lyapunov Functions}

In order to study the stability properties of the switched linear system (1) we assume that each individual subsystem admits such a piecewise linear Lyapunov function. The efficient computation of each Lyapunov function is very important for the application of the proposed methodology to practical hybrid systems. In the previous section, we described a class of piecewise linear functions induced by polyhedral sets that contain the origin. A Lyapunov function for each individual subsystem can be defined by computing a positively invariant polyhedral set for the subsystem. In the following, we briefly give the necessary background for the computation of these piecewise linear Lyapunov functions. First, we briefly describe an important class of systems for which positively invariant polyhedral sets and the corresponding Lyapunov functions can be computed by a similarity transformation [2]. In this case, the Lyapunov functions can be described using the infinity norm. Second, we outline an algorithm $[6,7]$ which can be used for the computation of general positively invariant polyhedral sets.

A class of linear systems for which such a Lyapunov function can be computed very efficiently is presented in [2]. Consider the system $x(t+1)=A x(t)$ where the eigenvalues of the matrix $A$ are located in the complex plane within the square defined by the vertices $(1,0),(0, i),(-1,0)$, and $(0,-i)$. Then, the following result is shown.

Corollary 1. [2]. If all the eigenvalues $\lambda_{i}=\mu_{i} \pm \sigma_{i}$ of the $n^{\text {th }}$ order linear system $x(t+1)=A x(t)$ are in the open square $\left|\mu_{i}\right|+\left|\sigma_{i}\right|<1$, then there exists a matrix $W \in \Re^{n \times n}$ with rankW $=n$ such that the polyhedral set $P=\left\{x \in \Re^{n}\right.$ : $\left.\|W x\|_{\infty}<1\right\}$ is a positively invariant set for the system.

The matrix $W$ can be computed as the solution to the matrix equation

$$
W A-Q W=0
$$

with the condition $\|Q x\|_{\infty}<1$. It is well known [10] that if the matrices $A$ and $Q$ do not have common eigenvalues then (8) has only the trivial solution $W=0$. 
The important assumption in Corollary 1 is that $W \in \Re^{n \times n}$ with $\operatorname{rank} W=n$. In this case, $W$ can be computed as the similarity transformation matrix by which $A$ is transformed to the Real Jordan Canonical Form [10].

We presented a class of discrete-time linear systems for which positively invariant polyhedral sets are described by the Lyapunov function $V(x)=\|W x\|_{\infty}$ and can be computed very efficiently. However, it should be noted that in our stability analysis for switched linear systems, it is not necessary for the individual invariant polyhedral sets to be centrally symmetric. Positively invariant polyhedral sets for stable discrete-time systems can be determined using computer generated Lyapunov functions [6]. The class of computer generated Lyapunov functions has been used for stability analysis of nonlinear systems in $[6,7,18$, 20]. The main idea is to construct a Lyapunov function that guarantees the stability of a set of matrices that is determined by applying Euler's discretization method to a system of nonlinear differential equations.

Our approach here is to use a computer generated Lyapunov function for each individual subsystem. Consider the matrix $A \in \Re^{n \times n}$ and let $P_{0} \subset \Re^{n}$ be a bounded polyhedral region of the origin. We denote the convex hull of $P$ by $\operatorname{conv}(P)$. Following $[6]$ we define

$$
P_{k}=\operatorname{conv}\left(\bigcup_{i=0}^{\infty} A^{i} P_{k-1}\right)
$$

and

$$
P^{*}=\bigcup_{i=0}^{\infty} P_{i} .
$$

The following results may be found in [6]: First, the matrix $A$ is stable if and only if $P^{*}$ is bounded. Second, if $A$ is stable then each set $P_{k}$ can be computed by $P_{k-1}$ using finitely many iterations. Furthermore, it is shown in [7] that if there exists constant $K \in \Re$ such that the eigenvalues of $A$ satisfy the condition $\left|\lambda_{i}\right| \leq K<1$, then the set $P^{*}$ is finitely computable. In this case the set $P^{*}$ is polyhedral as the convex hull of finitely many points. Furthermore, $P^{*}$ is a positively invariant set of the system. Then, a piecewise linear Lypunov function can be defined as the Lypunov function induced by the set $P^{*}$.

\section{$4 \quad$ Stabilizing Switching Sequences}

In this section, we present an approach based on multiple Lyapunov functions for the stability analysis of the switched system (1). The main contribution is an efficient characterization of a class of switching laws of the form (2) which guarantee the stability of the system. We assume that each individual subsystem admits a positively invariant polyhedral set that contains the origin which is described by

$$
P_{q}=\left\{x \in \Re^{n}: W^{q} x<\overline{1}\right\}
$$

where $W^{q} \in \Re^{m_{q} \times n}$ and $\overline{1}=[1, \ldots, 1]^{T} \in \Re^{n}$. In view of the above results, such a polyhedral set can be computed if the there exists constant $K \in \Re$ such that 
the eigenvalues of $A_{q}$ satisfy the condition $\left|\lambda_{i}\right| \leq K<1$. We denote the rows of the matrix $W^{q}$ by $w_{i}^{q} \in \Re^{n}, i=1, \ldots, m_{q}$. The Lyapunov function induced by the set $P_{q}$ can be described by

$$
V_{q}(x)=\max _{1 \leq i \leq m_{q}}\left\langle x, w_{i}^{q}\right\rangle .
$$

We consider a class $S$ of switching sequences that can be described by $s=$ $\left(q_{0}, t_{0}\right),\left(q_{1}, t_{1}\right), \ldots,\left(q_{j}, t_{j}\right), \ldots, x\left(t_{0}\right)=x_{0}$. It is assumed that if $s$ is finite then $t_{j+1}=\infty$ and that $q_{j} \neq q_{j+1}$. Such a sequence can be generated by the switching law $q_{j}\left(t_{j}+1\right)=\delta\left(q_{j-1}\left(t_{j}\right), x\left(t_{j}\right)\right), j=1,2, \ldots$

Proposition 1. Consider a switching sequence $s \in S$. If $V_{q_{j}}\left[x\left(t_{j}+1\right)\right] \leq V_{q_{j-1}}\left[x\left(t_{j}\right)\right]$, $j=1,2, \ldots$, then the switched system $x(t+1)=A_{q} x(t)$ is stable in the sense of Lyapunov.

Proof. Consider the multiple Lyapunov function defined by

$$
V[x(t)]=V_{q_{j}}[x(t)], \quad t_{j}<t \leq t_{j+1}
$$

then by the definition of $V_{q_{j}}$ we have that for every $t>t_{0}, t \in \mathbf{Z}^{+}$

$$
D V(x)=V[x(t+1)]-V[x(t)] \leq 0 .
$$

Note that the switched system for a fixed switching sequence $s$ can be viewed as a time-varying system. Since $V(x)$ is positive definite and radially unbounded, and $D V$ negative semidefinite, the system is stable in the sense of Lyapunov; see for example [1].

A multiple Lyapunov function composed by piecewise linear Lyapunov functions of the individual subsystems offers a significant advantage. It allows the characterization of the switching sequences that satisfy the condition of Proposition 1 by computing a conic partition of the state space.

First, we briefly describe the necessary notions and notation from convex analysis in order to construct the conic partition. Given a polytope $P \in \Re^{n}$, then a face of dimension $k$ is denoted as $k$-face $F$. The hyperplane that corresponds to a $k$-face $F$ is defined by the affine hull of $F$ and is denoted by aff $(F)$. Each $(n-1)$-face corresponds to a hyperplane that is defined by aff $\left(F_{i}\right)=\{x \in$ $\left.\Re^{n}:\left\langle x, w_{i}\right\rangle=1\right\}$ where $w_{i} \in \Re^{n}$ is the corresponding gradient vector. The set of vertices of $F$ can be found as $\operatorname{vert}(F)=\operatorname{vert}(P) \cap \operatorname{aff}(F)$ where $\operatorname{vert}(P)$ is the set of vertices of the polytope $P$. Finally, we denote the cone generated by the vertices of $F$ by cone $(F)$. Consider a pair of subsystems with matrices $A_{q_{1}}$ and $A_{q_{2}}$. We want to compute the region $\Omega_{q_{1}}^{q_{2}}=\left\{x \in \Re^{n}: V_{q_{2}}(x) \leq V_{q_{1}}(x)\right\}$. Consider the faces $F_{i_{1}}^{q_{1}}$ and $F_{i_{2}}^{q_{2}}$ of the polytopes $P_{q_{1}}$ and $P_{q_{2}}$ respectively and assume that $C=\operatorname{cone}\left(F_{i_{1}}^{q_{1}}\right) \cap \operatorname{cone}\left(F_{i_{2}}^{q_{2}}\right) \neq \emptyset$. Next, we define the halfspace $H_{q_{1}}^{q_{2}}=\left\{x \in \Re^{n}:\left\langle x, w_{i_{2}}^{q_{2}}-w_{i_{1}}^{q_{1}}\right\rangle \leq 0\right\}$ and the set $\Omega=C \cap H_{q_{1}}^{q_{2}}$. It is shown in the following lemma that the multiple Lyapunov function defined in Proposition 1 is decreasing if the system switches from $q_{1}$ to $q_{2}$ while $x \in \Omega$. 
Lemma 1. For every $x \in \Omega$ we have that $V_{q_{2}}(x) \leq V_{q_{1}}(x)$.

Proof. For every $x \in C$ the Lyapunov functions for the subsystems are given by $V_{q_{1}}(x)=\left\langle x, w_{i_{1}}^{q_{1}}\right\rangle$ and $V_{q_{2}}(x)=\left\langle x, w_{i_{2}}^{q_{2}}\right\rangle$ respectively. If $x \in \Omega$ we have that $\left\langle x, w_{i_{2}}^{q_{2}}-w_{i_{1}}^{q_{1}}\right\rangle \leq 0$ since $x \in H_{q_{1}}^{q_{2}}$, and therefore $V_{q_{2}}(x) \leq V_{q_{1}}(x)$.

Since $0 \in H_{q_{1}}^{q_{2}}$, the set $\Omega$ is a clearly a polyhedral cone as the intersection of cones with a common apex $(x=0)$ as shown in Fig. 2. The set $\Omega_{q_{1}}^{q_{2}}$ can be computed as the union of polyhedral cones by repeating the above procedure for all the pairs $\left(F_{i_{1}}^{q_{1}}, F_{i_{2}}^{q_{2}}\right)$ of $(n-1)$-faces of the polytope $P$ as shown in the following algorithm.

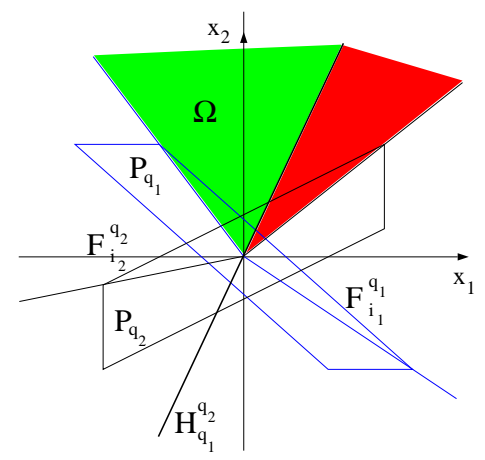

Fig. 2. The conic partition of the state space.

\section{Algorithm for the computation of $\Omega_{q_{1}}^{q_{2}}$}

INPUT: $W^{q_{1}}, W^{q_{2}}$;

for $i_{1}=1, \ldots, m_{q_{1}}$

for $i_{2}=1, \ldots, m_{q_{2}}$

$C=\operatorname{cone}\left(F_{i_{1}}^{q_{1}}\right) \cap \operatorname{cone}\left(F_{i_{2}}^{q_{2}}\right)$;

if $C \neq \emptyset$ then

$H_{q_{1}}^{q_{2}}=\left\{x \in \Re^{n}:\left\langle x, w_{i_{2}}^{q_{2}}-w_{i_{1}}^{q_{1}}\right\rangle \leq 0\right\}$

$\Omega=C \cap H_{q_{1}}^{q_{2}}$

$$
\Omega_{q_{1}}^{q_{2}}=\Omega_{q_{1}}^{q_{2}} \cup \Omega
$$

end

$$
\text { end }
$$

end

The above procedure can be repeated for every pair of subsystems to identify a class of stabilizing switching signals for the switched linear system. The class of switching sequences is characterized by the following result. Note that a numerical example that illustrates the approach may be found in [15]. 
Theorem 2. Consider the class of switching sequences $S$ defined by

$$
\begin{aligned}
q_{j}\left(t_{j}+1\right) & =\delta\left(q_{j-1}\left(t_{j}\right), x\left(t_{j}\right)\right) \\
x\left(t_{j}\right) & \in \Omega_{q_{j-1}}^{q_{j}} \neq \emptyset
\end{aligned}
$$

for $j=1,2, \ldots$. The switched linear system $x(t+1)=A_{q} x(t)$ is stable in the sense of Lyapunov for every switching sequence $s \in S$.

Proof. By induction, we have that if $s=\left(q_{0}, t_{0}\right)$ then the system is stable since $A_{q_{0}}$ is stable. Assume that the switched system is stable for the switching sequence $s=\left(q_{0}, t_{0}\right),\left(q_{1}, t_{1}\right), \ldots,\left(q_{j-1}, t_{j-1}\right)$ and consider the sequence $s^{\prime}=\left(q_{0}, t_{0}\right),\left(q_{1}, t_{1}\right), \ldots,\left(q_{j-1}, t_{j-1}\right),\left(q_{j}, t_{j}\right)$. Since $x\left(t_{j}\right) \in \Omega_{q_{j-1}}^{q_{j}}$, we have that $V_{q_{j}}\left[x\left(t_{j}\right)\right] \leq V_{q_{j-1}}\left[x\left(t_{j}\right)\right]$. Therefore, the multiple Lyapunov function defined by $V[x(t)]=V_{q_{j}}[x(t)], \quad t_{j}<t \leq t_{j+1}$ is decreasing for every $t$ and the system is stable in the sense of Lyapunov.

\section{Continuous-time Switched Linear Systems}

In this section, a characterization of stabilizing switching sequences for continuoustime switched linear systems is presented. The set of stabilizing switching sequences is characterized by computing a conic partition of the state space similarly to the discrete-time case. We consider the switched linear system

$$
\dot{x}(t)=A_{q} x(t), \quad q \in Q\{1, \ldots, N\}
$$

where $x(t) \in \Re^{n}$ and $A_{q} \in \Re^{n \times n}$. The switching law is described by

$$
q\left(t^{+}\right)=\delta(q(t), x(t)) .
$$

where $t^{+}=\lim _{\tau \rightarrow t, \tau>t} \tau$. The problem is to identify classes of switching signals generated by (18) for which the system (17) is stable. Note that in the following it is assumed that only finitely many switchings can occur in a finite time interval.

\subsection{Background Material}

In order to study the stability properties of the switched linear system (17), we assume that each individual subsystem admits a piecewise linear Lyapunov function induced by a positively invariant polyhedral set. Next, we summarize some results from [13] for the computation of piecewise linear Lyapunov functions for a class of continuous-time linear systems. Consider the continuous-time linear system $\dot{x}(t)=A x(t)$ where $x(t) \in \Re^{n}$ and $A \in \Re^{n \times n}$.

Similarly to the discrete-time case, there exists a class of continuous linear systems for which a positively invariant polyhedral set can be computed very efficiently. If the eigenvalues $\lambda_{i}$ of the linear system satisfy the condition $\left|\operatorname{Im}\left\{\lambda_{i}\right\}\right|<\left|\operatorname{Re}\left\{\lambda_{i}\right\}\right|$ then a Lyapunov function $V(x)=\|W x\|_{\infty}$ can be constructed using a similarity transformation [13]. 
The use of piecewise linear Lyapunov functions for the stability of linear systems is based on the following result [11]. Assume that there exists a function $V(x)$ such that $V$ is positive definite and radially unbounded, and the upper right Dini derivative [4] of $V$ satisfies the condition

$$
D V=\lim _{\Delta t \rightarrow 0} \sup \frac{V[x(t+\Delta t)]-V[x(t)]}{\Delta t}<0 .
$$

Then, the equilibrium $x=0$ is globally asymptotically stable.

The conditions for $V(x)=\|W x\|_{\infty}$ to be a Lyapunov function for the system $\dot{x}(t)=A x(t)$ can be stated using the logarithmic norm induced by the infinity norm. The logarithmic norm $\mu_{\infty}$ of a matrix $Q \in \Re^{n \times n}$ is defined as [9]

$$
\begin{aligned}
\mu_{\infty} & =\lim _{\alpha \rightarrow 0^{+}} \frac{\|I-\alpha Q\|_{\infty}-1}{\alpha} \\
& =\max _{i}\left\{q_{i i}+\sum_{j=1, j \neq i}\left|q_{i j}\right|\right\} .
\end{aligned}
$$

Theorem 3. [13] $V(x)=\|W x\|_{\infty}$ is a Lyapunov function for the system $\dot{x}=$ $A x(t)$ if and only if there exists $Q \in \Re^{n \times n}$ such that $W A-Q W=0$ and $\mu_{\infty}(Q)<0$.

Corollary 2. [13] If all the eigenvalues $\lambda_{i}=\mu_{i} \pm \sigma_{i}$ of the $n^{\text {th }}$ order system $\dot{x}=A x(t)$ satisfy the condition $\left|\mu_{i}\right| \leq\left|\sigma_{i}\right|$, then there exists $W \in \Re^{n \times n}$ with rankW $=n$ such that the polyhedral set $P=\left\{x \in \Re^{n}:\|W x\|_{\infty}<1\right\}$ is a positively invariant set for the system.

The above corollary is a consequence of the fact that the matrix equation $W A-Q A=0$ has a solution $W$ with $\operatorname{rank} W=n$ if and only if the eigenvalues of $A$ are identical with the eigenvalues of $Q$ [10]. The matrix $W$ can be computed as the similarity transformation matrix by which $A$ is transformed to the real Jordan canonical form similar to the discrete-time case.

\subsection{Stabilizing Switching Sequences}

In this section, we present an approach based on multiple Lyapunov functions for the stability analysis of the switched system (17). We assume that each individual subsystem admits a piecewise linear Lyapunov function described by the infinity norm. The main contribution is an efficient characterization of a class of switching laws of the form (2) which guarantee the stability of the system. Similar results can be developed for more general piecewise linear Lyapunov functions as in the discrete-time case in Section 4. We assume that each individual subsystem admits a positively invariant polyhedral set that contains the origin which is described by

$$
P_{q}=\left\{x \in \Re \Re^{n}:\left\|W^{q} x\right\|_{\infty}<1\right\}
$$


where $W^{q} \in \Re^{n \times n}$. We denote the rows of the matrix $W^{q}$ by $w_{i}^{q} \in \Re^{n}, i=$ $1, \ldots, n$. We consider a class $S$ of switching sequences that are described by $s=\left(q_{0}, t_{0}\right),\left(q_{1}, t_{1}\right), \ldots,\left(q_{j}, t_{j}\right), \ldots, x\left(t_{0}\right)=x_{0}$ where $t_{j} \in \Re^{n}, j=0,1, \ldots$ It is assumed that the sequence of switching instants $t_{0}, t_{1}, \ldots, t_{j}, \ldots$ is divergent in the sense that there are no infinitely many switchings in a finite time interval. Similarly to the discrete-time case, it is assumed that $q_{j} \neq q_{j+1}$. A sequence $s$ can be generated by the switching law $q_{j}\left(t_{j}^{+}\right)=\delta\left(q_{j-1}\left(t_{j}\right), x\left(t_{j}\right)\right), j=1,2, \ldots$.

Proposition 2. Consider a switching sequence $s \in S$. If $V_{q_{j}}\left[x\left(t_{j}^{+}\right)\right] \leq V_{q_{j-1}}\left[x\left(t_{j}\right)\right]$, $j=1,2, \ldots$, then the switched system $\dot{x}=A_{q} x(t)$ is stable in the sense of Lyapunov.

Proof. Consider the multiple Lyapunov function defined by

$$
V[x(t)]=V_{q_{j}}[x(t)], \quad t_{j}<t \leq t_{j+1} .
$$

Then, we have

$$
D V=\lim _{\Delta t \rightarrow 0} \sup \frac{V[x(t+\Delta t)]-V[x(t)]}{\Delta t} \leq 0 .
$$

for every $t \in \Re^{n}$ and therefore, the equilibrium $x=0$ is stable in the sense of Lyapunov; see for example [11].

A conic partition of the state space can be used to characterize a class of switching sequences that satisfy the condition of Proposition 2. Consider a pair of subsystems with matrices $A_{q_{1}}$ and $A_{q_{2}}$. The region $\Omega_{q_{1}}^{q_{2}}=\left\{x \in \Re^{n}: V_{q_{2}}(x) \leq\right.$ $\left.V_{q_{1}}(x)\right\}$ can be computed as a union of finitely generated cones and can be computed by the algorithm presented in Section 4 similarly to the discrete-time case. The class of stabilizing switching sequences is characterized by the following result.

Theorem 4. Consider the class of switching sequences $S$ defined by

$$
\begin{aligned}
q_{j}\left(t_{j}^{+}\right) & =\delta\left(q_{j-1}\left(t_{j}\right), x\left(t_{j}\right)\right) \\
x\left(t_{j}\right) & \in \Omega_{q_{j-1}}^{q_{j}} \neq \emptyset
\end{aligned}
$$

for $j=1,2, \ldots$ The switched linear system $\dot{x}=A_{q} x(t)$ is stable in the sense of Lyapunov for every switching sequence $s \in S$.

Proof. Similar to the proof of Theorem 2.

Example 1. Consider the switched discrete-time linear system

$$
\dot{x}=A_{q} x(t), \quad q \in\{1,2\}
$$

where

$$
A_{1}=\left[\begin{array}{cc}
1.7 & 1.8 \\
-4.5 & -3.7
\end{array}\right] \text { and } A_{2}=\left[\begin{array}{cc}
0.7 & -1 \\
1.6 & -1.7
\end{array}\right]
$$


The eigenvalues of the matrices $A_{1}$ and $A_{2}$ are $\lambda=-1 \pm .9 j$ amd $\lambda=-.5 \pm .4 j$. The real Jordan canonical form can be computed by the following similarity transformations.

$$
Q_{1}=W^{1} A_{1}\left(W^{1}\right)^{-1}=\left[\begin{array}{cc}
-1 & 0.9 \\
-0.9 & -1
\end{array}\right] \text { where } W^{1}=\left[\begin{array}{ll}
2 & 1 \\
1 & 1
\end{array}\right]
$$

and

$$
Q_{2}=W^{2} A_{2}\left(W^{2}\right)^{-1}=\left[\begin{array}{cc}
-0.5 & 0.4 \\
-0.4 & -0.5
\end{array}\right] \text { where } W^{2}=\left[\begin{array}{cc}
-1 & 1 \\
1 & -0.5
\end{array}\right] .
$$

We have that $\mu_{\infty}\left(Q_{1}\right)=-0.1<0$ and therefore, $V_{1}(x)=\left\|W^{1} x\right\|_{\infty}$ is a Lyapunov function for the subsystem $A_{1}$. Similarly, $\mu_{\infty}\left(Q_{2}\right)=-0.1<0$ and $V_{2}(x)=\left\|W^{2} x\right\|_{\infty}$ is a Lyapunov function for the subsystem $A_{2}$. The functions $V_{1}$ and $V_{2}$ correspond to the positively invariant polyhedral sets

$$
P_{1}=\left\{x \in \Re^{2}:\left\|W^{1} x\right\|_{\infty} \leq 1\right\} \text { and } P_{2}=\left\{x \in \Re^{2}:\left\|W^{2} x\right\|_{\infty} \leq 1\right\}
$$

shown in Fig. 3(i).
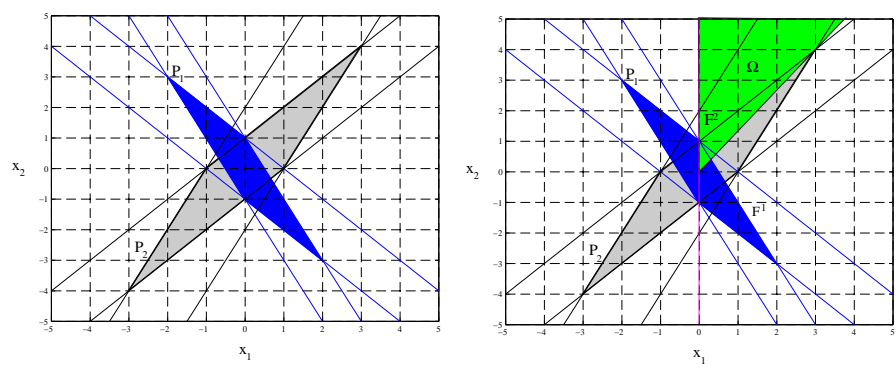

Fig. 3. (i) Positively invariant polyhedral sets, (ii) The region $\Omega$.

Consider the faces $F^{1}$ and $F^{2}$ shown in Fig. 3(ii). For every $x \in \operatorname{cone}\left(F^{1}\right) \cap$ cone $\left(F^{2}\right)$ we have that $V_{1}(x)=\left\langle x, w^{1}\right\rangle$ and $V_{2}(x)=\left\langle x, w^{2}\right\rangle$ with $w^{1}=[2,1]$ and $w^{2}=[-1,1]$ respectively. We consider the halfspace

$$
\begin{aligned}
H_{1}^{2} & =\left\{x \in \Re^{2}:\left\langle x, w^{2}-w^{1}\right\rangle \leq 0\right\} \\
& =\left\{x \in \Re^{2}: x_{1} \geq 0\right\} .
\end{aligned}
$$

Therefore, for every $x \in \Omega=\operatorname{cone}\left(F^{1}\right) \cap \operatorname{cone}\left(F^{2}\right) \cup H_{1}^{2}$ we have that $V_{2}(x) \leq$ $V_{1}(x)$.

By repeating the procedure for all the pairs of faces for the polytopes $P_{1}$ and $P_{2}$ the we compute the region

$$
\begin{aligned}
\Omega_{q_{1}}^{q_{2}} & =\left\{x \in \Re^{2}: V_{q_{2}}(x)<V_{q_{1}}(x)\right\} \\
& =\left\{x \in \Re^{2}: x_{1}>0\right\} .
\end{aligned}
$$


Similarly we have that

$$
\begin{aligned}
\Omega_{q_{2}}^{q_{1}} & =\left\{x \in \Re^{2}: V_{q_{1}}(x)<V_{q_{2}}(x)\right\} \\
& =\left\{x \in \Re^{2}: x_{1}<0\right\} .
\end{aligned}
$$

Therefore, for any switching sequence $s$ given by the switching law

$$
\begin{aligned}
q_{2}\left(t^{+}\right) & =\delta\left(q_{1}(t), x(t)\right) \\
x(t) & \in \Omega_{q_{1}}^{q_{2}}
\end{aligned}
$$

and

$$
\begin{aligned}
q_{1}\left(t^{+}\right) & =\delta\left(q_{2}(t), x(t)\right) \\
x(t) & \in \Omega_{q_{2}}^{q_{1}}
\end{aligned}
$$

the switched system is stable. A stable trajectory is shown in Fig. 4(i).
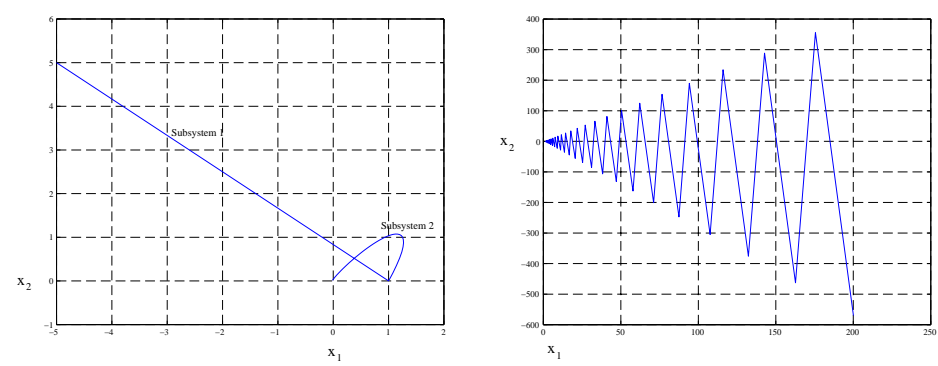

Fig. 4. (i) A stable trajectory. (ii) An unstable trajectory.

The characterization of the stabilizing switching sequences is based on sufficient conditions. Therefore, for a switching sequence $s$ that does not satisfy the formulated conditions, the switched system is not necessarily unstable. However, the switched system (27) can generate unstable trajectories as shown in Fig. 4(ii). An unstable trajectory can be generated by requiring that the system will keep switching indefinitely and that the Lyapunov function is increasing at every switching.

\section{Conclusions}

In this paper, a class of stabilizing switching sequences for switched linear systems is characterized by computing conic partitions of the state space. The main advantage of the approach is that the methodology for computing switching laws that guarantee stability is based on the parameters of the system and so, trajectories for particular initial conditions do not need to be calculated. Therefore, the proposed approach can be used very efficiently to investigate the stability properties of practical hybrid systems. 


\section{References}

1. P. Antsaklis and A. Michel. Linear Systems. McGraw-Hill, 1997.

2. G. Bitsoris. Positively invariant polyhedral sets of discrete-time linear systems. International Journal of Control, 47(6):1713-1726, 1988.

3. F. Blanchini. Nonquadratic Lyapunov functions for robust control. Automatica, 31(3):451-461, 1995.

4. F. Blanchini. Set invariance in control. Automatica, 35(11):1747-1767, 1999.

5. M. Branicky. Multiple Lyapunov functions and other analysis tools for switched and hybrid systems. IEEE Trans. on Automatic Control, 43(4):475-482, 1998.

6. R. Brayton and C. Tong. Stability of dynamical systems: A constructive approach. IEEE Trans. on Circuits and Systems, CAS-26(4):224-234, 1979.

7. R. Brayton and C. Tong. Constructive stability and asymptotic stability of dynamical systems. IEEE Trans. on Circuits and Systems, CAS-27(11):1121-1130, 1980.

8. R. DeCarlo, M. Branicky, S. Pettersson, and B. Lennartson. Perspectives and results on the stability and stabilizability of hybrid systems. Proceedings of IEEE, 88(7):1069-1082, July 2000.

9. V. Desoer and H. Haneda. The measure of a matrix as a tool to analyze computer algorithms for circuit analysis. IEEE Trans. on Circuit Theory, 19(5):480-486, 1972.

10. F. Gantmacher. Matrix Theory. Chelsea, 1959.

11. W. Hahn. Stability of Motion. Springer-Verlag, 1967.

12. M. Johansson and A. Rantzer. Computation of piecewise quadratic Lyapunov functions for hybrid systems. IEEE Trans. on Automatic Control, 43(4):555-559, 1998.

13. H. Kiendl, J. Adamy, and P. Stelzner. Vector norms as Lyapunov functions for linear systems. IEEE Trans. on Automatic Control, 37(6):839-1842, 1992.

14. X. Koutsoukos. Analysis and Design of Piecewise Linear Hybrid Dynamical Systems. PhD thesis, Department of Electrical Engineering, University of Notre Dame, Notre Dame, IN, 2000.

15. X. Koutsoukos and P. Antsaklis. Stabilizing supervisory control of hybrid systems based on piecewise linear Lyapunov functions. In Proceedings of the 8th IEEE Mediterranean Conference on Control and Automation, Rio, Greece, July 2000.

16. D. Liberzon and A. Morse. Basic problems in stability and design of switched systems. IEEE Control Systems Magazine, 19(5):59-70, October 1999.

17. A. Michel. Recent trends in the stability analysis of hybrid dynamical systems. IEEE Trans. on Circuits and Systems I, 46(1):120-134, 1999.

18. A. Michel, B. Nam, and V. Vittal. Computer generated Lyapunov functions for interconnected systems: Improved results with applications to power systems. IEEE Trans. on Circuits and Systems, CAS-31(2):189-198, 1984.

19. A. Molchanov and Y. Pyatnitskiy. Criteria of asymptotic stability of differential and difference inclusions encountered in control theory. Systems $\&$ Control Letters, 13:59-64, 1989.

20. Y. Ohta, H. Imanishi, L. Gong, and H. Haneda. Computer generated Lyapunov functions for a class of nonlinear systems. IEEE Trans. on Circuits and Systems-I: Fundamental Theory and Applications, 40(5):428-433, 1993.

21. S. Pettersson and B. Lennartson. Stability and robustness of hybrid systems. In Proceedings of the 35th IEEE Conference on Decision and Control, pages 1202-1207, Kobe, Japan, December 1996.

22. H. Ye, A. Michel, and L. Hou. Stability theory for hybrid dynamical systems. IEEE Trans. on Automatic Control, 43(4):461-474, 1998. 